\title{
Temperature-Dependent Thermoelastic Anisotropy of the Phenyl Pyrimidine Liquid Crystal
}

 \\ ${ }^{\dagger}$ School of Materials and Chemical Technology and ${ }^{\S}$ Tokyo Tech World Research Hub Initiative (WRHI), School of Materials and \\ Chemical Technology, Tokyo Institute of Technology, Ookayama, Meguro-ku, Tokyo 152-8550, Japan \\ ${ }^{\ddagger}$ Max Planck Institute for Polymer Research, Ackermannweg 10, 55128 Mainz, Germany
}

Supporting Information

ABSTRACT: Controlling thermoelastic anisotropy of liquid crystals (LCs) is important for achieving reliable structural stability and efficient heat dissipation, especially for high-performance LC devices. A solid understanding of the thermoelastic anisotropy and its relation with the LC molecular structure is, however, still missing. Here, we studied the direction-dependent mechanical and thermal properties of 5- $n$-octyl-2-(4- $n$-octyloxy-phenyl)-pyrimidine (PYP8O8) in a wide temperature range, covering five phases (i.e., crystalline, smectic C, smectic $A$, nematic, and liquid), by Brillouin light spectroscopy and temperature wave analysis, respectively. We found that the mechanical anisotropy is much smaller than the thermal anisotropy at LC phases; both anisotropies show strong phase dependence,


with the biggest change occurring at the crystalline to LC phase transition; and the anisotropy of the phonon mean-free path correlates with the structural anisotropy of the rigid core of the LC molecule. The analysis of the temperature-dependent thermoelastic anisotropy of LCs yields insights into structure-based phonon engineering.

\section{INTRODUCTION}

Liquid crystals (LCs) are remarkable materials that exhibit crystal-like and/or liquid-like behaviors depending on the operating temperature. ${ }^{1}$ As temperature increases, a typical LC displays phase transitions from a solid phase to LC phases (e.g., smectic C, smectic A, nematic) and finally to a liquid phase. ${ }^{1}$ The easy temperature tunability between different phases and the unique properties in each phase have led LCs into many applications, such as flat panel displays, organic electronics, and LC elastomer actuators. ${ }^{2}$ LCs are inherently, mechanically, and thermally anisotropic because of the rodshaped molecules. The ever increasing packing density and operating frequencies of LC devices ${ }^{3}$ require stringently controlling the thermoelastic anisotropy of LCs so as to achieve reliable structural stability and efficient heat dissipation.

Abundant research work has been carried out to understand the thermoelastic anisotropy of LCs and how it is related to the molecular structure. ${ }^{4-22}$ For mechanical anisotropy, it is generally accepted that (1) the elastic constants $(C)$ are higher along $(I I)$ the director than perpendicular $(\perp)$ to it, (2) the elastic anisotropies, $\left(C_{\|}-C_{\perp}\right) / C_{\perp}$, is on the order of a few percent or less, and (3) the dimensional ratio of rigid cores and side chains determines the elastic anisotropy. ${ }^{4-11}$ For thermal anisotropy, it has been shown that (1) the thermal diffusivity $(\alpha)$ is higher along the director than perpendicular to it, (2) the thermal anisotropy, $\left(\alpha_{\|}-\alpha_{\perp}\right) / \alpha_{\perp}$, could reach $100 \%$ or even more, and (3) the thermal anisotropy stems from structural anisotropy of the LC molecules, and is not dependent on the long-range layer ordering of the LC molecules. $^{5-22}$ However, previous research typically focused on either the mechanical or the thermal properties in 1-2 phases of the LCs. To the best of our knowledge, no combined study of the anisotropic mechanical and thermal properties of LCs over a wide range of temperatures has been performed. This kind of studies, however, is crucial to gain a well-rounded understanding of the direction-dependent phonon transport in LCs, as mechanical and thermal properties involve different bands in the phonon spectrum.

Thermal transport in dielectric materials is usually described by phonons over a range of mean-free paths (MFPs). In the kinetic theory, the thermal diffusivity, $\alpha$, is related to the phonon group velocity, $v$, and phonon $\operatorname{MFP}, \Lambda$, as ${ }^{23}$

$$
\alpha=\sum_{s} \int_{0}^{\infty} v(\omega) \Lambda(\omega) \mathrm{d} \omega
$$

where $s$ is the polarization index, and $\omega$ is the angular phonon frequency. The MFP of a phonon represents an average distance between two consecutive phonon scattering events. Using effective properties, eq 1 could be rewritten in another useful form

$$
\alpha=\frac{1}{3} v_{\text {eff }} \Lambda_{\text {eff }}
$$

where the subscript "eff" indicates an effective property. Note $\alpha=k / C_{V}$, where $k$ is thermal conductivity, and $C_{V}$ is

Received: May 6, 2019

Revised: June 16, 2019

Published: July 9, 2019 


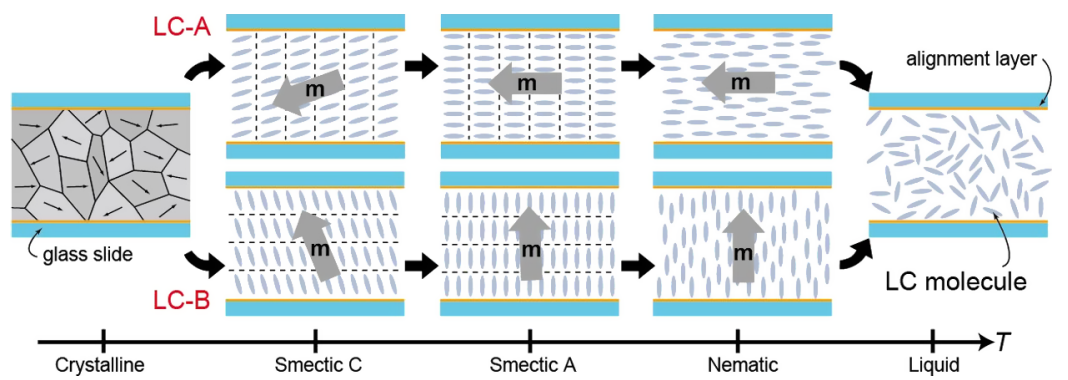

Figure 1. Schematic view (cross section) of the two types of LC film samples (LC-A and LC-B) used in this study. The LC films are sandwiched between two glass slides, with the alignment of the LC molecules (indicated by $\mathbf{m}$ ) controlled by a thin surfactant layer. In the $\mathrm{SmC}$, SmA, and $\mathrm{N}$ phases, LC-A and LC-B feature molecule alignments parallel and perpendicular to the glass slides, respectively. In the crystalline phase, both LC-A and LC-B are polycrystalline. In the liquid phase, the LC molecules are randomly oriented in both LC-A and LC-B.

volumetric specific heat. It is seen that $\alpha$ is correlated with $v_{\text {eff }}$ via $\Lambda_{\text {eff }}$ In the kinetic model, the phonon group velocity is typically assumed to be the sound velocity. Because sound velocity is directly related to elasticity, eq 2 indicates that $\Lambda_{\text {eff }}$ could be used to characterize the relationship between the thermal and mechanical properties. For an anisotropic material like a LC, a weak (strong) direction dependency in $\Lambda_{\text {eff }}$ implies similar (distinct) thermal and mechanical anisotropies. As thermoelastic anisotropy stems from the underlying structural anisotropy, a better understanding of the former holds promises for improved material designs through engineering of the latter.

In this work, we investigate the anisotropic mechanical and thermal properties of a phenyl pyrimidine LC (PYP8O8) over a wide range of temperatures. PYP8O8 is chosen for the study because it exhibits five well-separated phases (i.e., crystalline, smectic C, smectic A, nematic, and isotropic liquid) in a manageable temperature range (e.g., $20-90^{\circ} \mathrm{C}$ ). The long side chains positioned on opposite sides of the rigid core also increase the symmetry of the LC samples, thus simplifying the data analysis. We utilize the Brillouin light spectroscopy (BLS) to probe hypersonic phonon propagating at different directions, which allows us to determine the complete elastic constants and moduli using a continuum mechanics model. We employ the temperature wave analysis (TWA) method to measure the thermal diffusivities of PYP8O8 in the directions parallel and perpendicular to the LC director. The simultaneous availability of the anisotropic mechanical and thermal properties further enables examination of their correlations in the different phases of PYP8O8 and the characteristic length scales for the anisotropic phonon transport in PYP8O8 in terms of the phonon MFP.

\section{EXPERIMENTAL SECTION}

Materials. We used LC samples made of 5-n-octyl-2-(4-noctyloxy-phenyl)-pyrimidine (PYP8O8) molecules $\left(\mathrm{C}_{26} \mathrm{H}_{40} \mathrm{~N}_{2} \mathrm{O}\right)$ in this study. The LC molecule contains a side chain with eight carbon atoms on both sides of the rigid phenyl pyrimidine core. The LC samples are sandwiched between two parallel glass slides, and have a thickness about $30 \mu \mathrm{m}$. The alignment of the LC molecules relative to the glass slides was controlled by a thin polyimide-type alignment layer coated on the glass slides. For samples used in the TWA experiments, a thin $\mathrm{Au}$ electrode was sputtered on the glass glides before the alignment layer was coated. Two types of alignment layers, AL1254 (JSR) and JALS204 (JSR), were used to prepare LC samples with the director (denoted by a vector $\mathbf{m}$ ) of the LC molecules oriented parallel (sample LC-A) and perpendicular (sample LC-B) to the glass slides, respectively (Figure 1). To quantify the anisotropic thermoelastic properties of the LC samples, we define two characteristic directions, $\| \mathbf{m}$ and $\perp \mathbf{m}$, which represent directions parallel and perpendicular to $\mathbf{m}$, respectively. The BLS and TWA experiments were conducted under atmospheric pressure, but the temperature was varied from 20 to $90{ }^{\circ} \mathrm{C}$. As temperature increases, the LC samples undergo the following phase sequence: Crys-29-SmC-56-SmA62-N-69-Iso, where the numbers indicate the transition temperatures (in ${ }^{\circ} \mathrm{C}$ ) between the adjacent LC phases (Crys: crystalline solid; SmC: smectic-C LC; SmA: smectic-A LC; N: nematic LC; and Iso: isotropic liquid). In the crystalline phase, both LC-A and LC-B are polycrystalline.

Brillouin Light Spectroscopy. High-resolution BLS measurements of the ordered films were performed using a six-pass tandem Fabry-Perot interferometer in conjunction with a Nd/YAG laser $(\lambda=532 \mathrm{~nm})$ mounted on a goniometer, allowing for angle-dependent experiments. The interaction between the incident light and thermally activated phonons leads to inelastic light scattering at a selected phonon wave vector $\mathbf{q}=\mathbf{k}_{\mathrm{i}}-\mathbf{k}_{\mathrm{s}}$, where the wave vectors of the incident $\left(\mathbf{k}_{\mathrm{i}}\right)$ and scattered light $\left(\mathbf{k}_{\mathrm{s}}\right)$ define the scattering plane. The BLS spectrum consists of a doublet around the central (elastic) Rayleigh line at frequencies, $f= \pm \frac{v}{2 \pi} q$, where $v$ is the sound velocity for homogeneous films. The experiments of LC-A were conducted in the transmission geometry, which allows probing phonons propagating parallel to the scattering plane with the phonon wave vector amplitude $q=\frac{4 \pi}{\lambda} \sin (\theta / 2)$ being independent of the refractive index $n$, where $\theta$ is the scattering angle. The longitudinal (transverse) phonon could be selectively observed in the $\mathrm{VV}(\mathrm{VH})$ spectra. The $\mathrm{VV}(\mathrm{VH})$ denotes the combined polarization of the incident and scattering light, selected by the input polarizer (V) and output analyzer ( $\mathrm{V}$ or $\mathrm{H}) ; \mathrm{V}(\mathrm{H})$ denotes vertically(horizontally) polarized light with respect to the scattering plane defined by the wave vectors of the incident and scattered light. For temperature-scan experiments, the temperature increases at a slow speed of $0.1{ }^{\circ} \mathrm{C} / \mathrm{min}$ and BLS spectra were collected after an isothermal equilibration of the whole setup for 10-20 min. The uncertainty of the sound velocities is estimated to be $1-$ $2 \%$.

Temperature Wave Analysis. TWA measurements ${ }^{18-22}$ were conducted to measure the thermal diffusivity of the LC samples in the $\| \mathbf{m}$ and $\perp \mathbf{m}$ directions. In a typical TWA experiment, sinusoidal Joule heating was introduced at the electrode on the bottom surface of a LC sample film to generate a temperature wave that propagated across the sample 


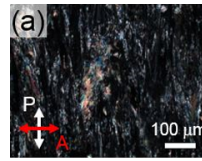

(b) $m$



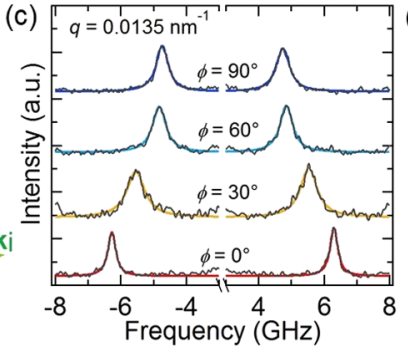

Figure 2. (a) Polarized optical microscopy image of LC-A in the crystalline phase. (b) Transmission scattering geometry. $\theta$ is the scattering angle [i.e., the angle between the incident $\mathbf{k}_{\mathrm{i}}$ and scattering $\mathbf{k}_{\mathrm{s}}$ wave vectors (in green)]. The in-plane phonon wave vector, $\mathbf{q}=\mathbf{k}_{\mathrm{s}}-\mathbf{k}_{\mathrm{i}}(\mathrm{red})$, forms an adjustable angle $\varphi$ with the LC director, $\mathbf{m}$. (c) VV-polarized BLS spectra of the polycrystalline LC-A recorded at $q=0.0135 \mathrm{~nm}^{-1}$ at four $\varphi$ angles. The spectra are represented by a single-Lorentzian peak (colored lines). (d) Longitudinal (red) and transverse (black and blue) sound velocities as a function of $\varphi$ at $T=21^{\circ} \mathrm{C}$. The solid lines are best fits based on a continuum mechanics model.

film. The phase delay of the temperature waves $(\Delta \xi(T))$ at the bottom and top surfaces of the LC sample was measured with a $120 \mathrm{~Hz}$ temperature wave. Together with the sample thickness (d), $\Delta \xi(T)$ was subsequently used to calculate the thermal diffusivity $(\alpha)$ according to $\Delta \xi(T)=-\sqrt{\frac{\omega_{\mathrm{T}}}{2 \alpha}} d-\frac{\pi}{4}$. Here, $\omega_{\mathrm{T}}$ is the angular frequency of the temperature wave. We assumed a one-dimensional heat flow, which was experimentally realized using a one-dimensional arrangement of the input and delayed temperature waves in the cross-plane direction of the sample. We measured both LC-A and LC-B to obtain the thermal diffusivities, $\alpha_{\|}$and $\alpha_{\perp}$, in the $\| \mathbf{m}$ and $\perp \mathbf{m}$ directions, respectively. The temperature-dependent TWA experiments were performed from 90 to $20^{\circ} \mathrm{C}$, at a cooling rate of $0.2{ }^{\circ} \mathrm{C}$ / min. The thermal diffusivity measurements were not conducted in the crystalline states of the LC samples because the samples become polycrystalline and thus it is impossible to resolve the direction-dependent thermal diffusivities. The uncertainty (confidence level 95\%) of the measured thermal diffusivities is estimated to be less than $5 \%$.

\section{RESULTS AND DISCUSSION}

Phonon Propagation and Mechanical Anisotropy in the Crys Phase. We first examine the phonon propagation in the crystalline phase of the PYP8O8 at room temperature $(T=$ $21{ }^{\circ} \mathrm{C}$ ). The noncontact and nondestructive BLS technique is employed to probe the hypersonic phonon propagation in LC$\mathrm{A}$ and hence the elastic moduli. ${ }^{24,25}$ Figure la shows a schematic of the LC-A film with the director $\mathbf{m}$ predominantly aligned parallel to the glass slides, as confirmed by polarized optical microscope image (Figure 2a). We utilized the transmission scattering geometry shown in Figure $2 b$ to direct the scattering wave vector $q$ parallel to the LC sample film. By rotating the LC-A crystal around the normal to the film, the angle $\varphi$ between $\mathbf{q}$ and $\mathbf{m}$ (Figure $2 \mathrm{~b}$ ) could be tuned in the range from $0^{\circ}$ to $90^{\circ}$. Figure $2 \mathrm{c}$ displays exemplary $\mathrm{VV}$ polarized BLS spectra at varying $\phi$ angles but at a constant $q=$ $0.0135 \mathrm{~nm}^{-1}$. The frequency $f$ of the BLS doublet, assigned to the longitudinal mode, depends on the direction of $\mathbf{q}$, implying a $\varphi$-dependent effective sound velocity, $v=\frac{2 \pi f}{q}$. In contrast, for an isotropic film with a direction-independent sound velocity, the position of the BLS doublet depends only on the magnitude of $\mathbf{q}$, not its direction. In fact, the longitudinal sound velocity, $v_{\mathrm{L}}$, exhibits strong $\varphi$-dependence as shown in Figure 2d. At $\varphi=0^{\circ}(\mathbf{q} \| \mathbf{m}), v_{\mathrm{L}}$ assumes its maximum $(2.98 \pm$ $0.03 \mathrm{~km} / \mathrm{s})$, while its minimum $(2.20 \pm 0.03 \mathrm{~km} / \mathrm{s})$ is attained at $\varphi=90^{\circ}(\mathbf{q} \perp \mathbf{m})$. Therefore, the sound speed in the $\| \mathbf{m}$ direction is about $35 \%$ higher than that in the $\perp \mathbf{m}$ direction. The experimental sound velocities measured at $\mathrm{GHz}$ frequencies $\left(\omega \approx 3 \times 10^{10} \mathrm{rad} / \mathrm{s}\right)$ represent the high frequency limit $(\omega \tau)^{2} \gg 1$, as the structural relaxation time, $\tau$, obtained by dielectric spectroscopy, is on the order of $1 \mathrm{~ns}$ in the temperature range $(20-50){ }^{\circ} \mathrm{C}$. Hence, the reported moduli in this work are free of viscoelastic effects. ${ }^{26}$ The angle-dependent sound velocities of LC-A in Figure $2 \mathrm{~d}$ suffice for determining the anisotropic mechanical properties of PYP8O8. The LC-B sample film is shown in Figure S1. For this sample, the BLS spectrum at a given wave vector reveals two acoustic phonons because LC-B features domain sizes larger than the probed phonon wavelength in the polycrystalline state. The analysis of the anisotropic phonon propagation in the LC-A sample helps identify the observed modes in the polycrystalline LC-B. The two resolved in the weak depolarized $(\mathrm{VH})$ spectrum were assigned to a pure-transverse $\left(\mathrm{T}_{1}\right)$ mode at $\varphi=-90^{\circ}$ and quasi-transverse $\left(\mathrm{T}_{2}\right)$ modes at $\varphi=-90^{\circ}$ and $0^{\circ}$.

It is well known that sound velocities are intimately related to the elastic tensor in the framework of the Christoffel equation. Given appropriate direction-dependent sound velocities, the elastic tensor could be determined via leastsquare fitting. The LC samples considered in this study could be assumed transversely isotropic, with the symmetry axis being parallel to $\mathbf{m}$. For the transversely isotropic material, there are five independent elastic stiffness constants: $C_{11}, C_{13}$, $C_{33}, C_{44}$, and $C_{66}{ }^{27}$ To facilitate the analysis, we construct a " 123 " coordinate system, with the " 3 -axis" being parallel to $\mathbf{m}$. For sound propagating in the direction forming an angle, $\varphi$, with the 3-axis, the sound velocities of three modes (the pure transverse mode $\left(\mathrm{T}_{1}\right)$, the quasi-longitudinal mode $(\mathrm{L})$, and the quasi-transverse mode $\left(\mathrm{T}_{2}\right)$ can be expressed as ${ }^{27}$

$$
\begin{aligned}
& v_{\phi, \mathrm{T}_{1}}=\sqrt{\frac{A_{1}}{\rho}} \\
& v_{\phi, \mathrm{L}}=\sqrt{\frac{-A_{2}+\sqrt{A_{2}^{2}-4 A_{3}}}{2 \rho}} \\
& v_{\phi, \mathrm{T}_{2}}=\sqrt{\frac{-A_{2}-\sqrt{A_{2}^{2}-4 A_{3}}}{2 \rho}}
\end{aligned}
$$

where

$$
A_{1}=\sin ^{2} \phi C_{66}+\cos ^{2} \phi C_{44}
$$


Table 1. Mechanical Properties of LC-A in the Crystalline Phase

\begin{tabular}{|c|c|c|c|c|c|}
\hline \multicolumn{2}{|c|}{ Young's modulus } & \multicolumn{2}{|c|}{ shear modulus } & \multicolumn{2}{|c|}{ Poisson's ratio } \\
\hline$E_{\|}(\mathrm{GPa})$ & $E_{\perp}(\mathrm{GPa})$ & $G_{23}=G_{13}(\mathrm{GPa})$ & $G_{12}(\mathrm{GPa})$ & $v_{23}=v_{13}$ & $v_{12}$ \\
\hline $8.5 \pm 0.1$ & $2.3 \pm 0.2$ & $1.64 \pm 0.06$ & $0.64 \pm 0.02$ & $0.02 \pm 0.04$ & $0.75 \pm 0.03$ \\
\hline
\end{tabular}

$$
\begin{aligned}
A_{2}= & -\left(\sin ^{2} \phi C_{11}+\cos ^{2} \phi C_{33}+C_{44}\right) \\
A_{3}= & \sin ^{4} \phi C_{11} C_{44}+\sin ^{2} \phi \cos ^{2} \phi\left(C_{11} C_{33}-C_{13}{ }^{2}-2 C_{13} C_{44}\right) \\
& +\cos ^{4} \phi C_{33} C_{44}
\end{aligned}
$$

Through nonlinear least-squared fitting of experimental sound velocities (Figure 2d) with the PYP8O8 density of 1.01 $\mathrm{g} / \mathrm{cm}^{3}{ }^{28}$ we obtained the elastic stiffness constants (eq 9). The elastic stiffness constants allow theoretical representation of the direction-dependent sound velocities (solid lines in Figure 2d). Furthermore, they can be used to calculate the engineering moduli and Poisson's ratios, ${ }^{27}$ as summarized in Table 1 . Note that $C_{13}$ is essentially zero within the experimental error and that the error of $C_{66}$ is $0.02 \mathrm{GPa}$.

$$
\begin{aligned}
& {\left[C_{i j}\right]=} \\
& {\left[\begin{array}{lllllll}
5.1 \pm 0.1 & 3.8 \pm 0.1 & 0.2 \pm 0.3 & & & \\
3.8 \pm 0.1 & 5.1 \pm 0.1 & 0.2 \pm 0.3 & & & \\
0.2 \pm 0.3 & 0.2 \pm 0.3 & 8.5 \pm 0.1 & & & \\
& & & 1.6 \pm 0.1 & & \\
& & & & 1.6 \pm 0.1 & \\
& & & & & 0.6 \pm 0.0
\end{array}\right]}
\end{aligned}
$$

The Young modulus $E_{\|}$is comparable to that of glassy polymers, but the material is much (73\%) softer in the normal direction. The shear modulus $G_{12}$ normal to the director (" 3 "axis) is rather low compared to $G_{13}$ along the director axis, which has a glassy polymer-like value (1-2 GPa). The Poisson's ratio is expectedly anisotropic with a solid-like behavior $v_{13} \approx 0$, implying robust cross-section upon stretching along the director axis. In the plane normal to the director, the Poisson's ratio exceeds the upper limit (0.5) for isotropic materials. However, for anisotropic materials, like the LC sample considered in the study, the Poisson's ratio is essentially unbounded. ${ }^{29}$ The large $v_{12}$ indicates a large response of the strain in the " 2 " direction upon a strain in the " 1 " direction.

Phonon Propagation and Mechanical Anisotropy in the LC Phases. The phonon propagation and mechanical anisotropy were also examined at higher temperatures to explore the thermomechanical behavior of PYP8O8 in the different LC phases. Because the effective sound velocities change their angle dependencies at around $\varphi=45^{\circ}$ (Figure $2 \mathrm{~d})$, the temperature dependency of the sound velocity was measured for $\varphi=0^{\circ}, 45^{\circ}$, and $90^{\circ}$ at the transmission scattering geometry (Figure $2 \mathrm{~b}$ ). Figure $3 \mathrm{a}$ depicts this variation for LC-A with the effective sound velocity assuming the lowest value for $\varphi=45^{\circ}$. Because of thermal expansion and/or change of the cohesive forces, the sound velocity for the three $\mathbf{q}$ directions decreases with increasing temperature as the material changes its phase (i.e., $\mathrm{SmC} \rightarrow \mathrm{SmA} \rightarrow \mathrm{N} \rightarrow$ Iso). The largest drop of sound velocity occurs at the first order Crys $\rightarrow$ SmC phase transition, and is stronger in the $\| \mathbf{m}$ direction $(\sim 50 \%)$ than the $\perp$ m direction $(\sim 30 \%)$ (inset to Figure $3 \mathrm{a})$. These results suggest that the large-scale periodicity like a lattice structure in a crystalline state is
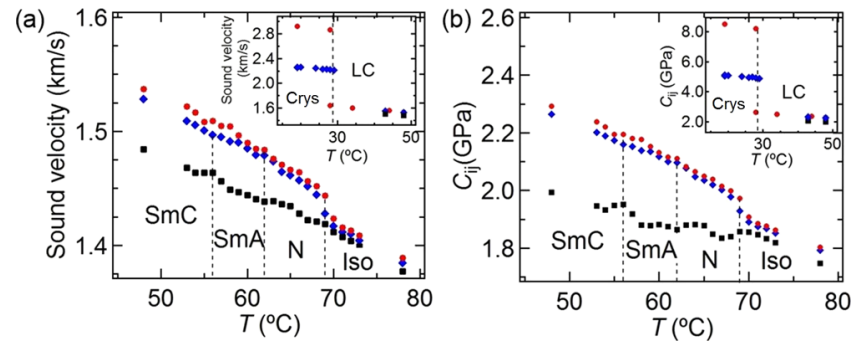

Figure 3. Temperature-dependent mechanical properties of LC-A. (a) Temperature dependency of the longitudinal sound velocities at $\varphi=$ $0^{\circ}$ (red), $45^{\circ}$ (blue), and $90^{\circ}$ (black). The vertical dashed lines indicate the LC phase transition temperatures. The inset shows the data in the crystalline and $\mathrm{SmC}$ phases. (b) Elastic stiffness constants, $C_{11}$ (blue diamond), $C_{33}$ (red circle), and $C_{13}$ (black square), as a function of temperature. Inset: Temperature dependency of the elastic constants, $C_{11}$ (blue diamond), $C_{33}$ (red circle), and $C_{13}$ (black square) in the crystalline and $\mathrm{SmC}$ phases.

critical for achieving strongly direction-dependent sound velocities.

Similar to the analysis performed to the BLS data of the LCA sample in the Crys phase, the sound velocities at $\varphi=0^{\circ}, 45^{\circ}$, and $90^{\circ}$ in the LC phases of LC-A could be used to deduce the elastic stiffness constants. Because the LC phases do not support shear stress and thus transverse sound propagation, it can be assumed that $C_{44} \approx 0$, and $C_{66} \approx 0$. Based on eqs 4,7 , and 8 , we obtain

$$
\begin{aligned}
& v_{\phi, \mathrm{L}}= \\
& {\left[\left(\left(\sin ^{2} \phi C_{11}+\cos ^{2} \phi C_{33}\right)+\right.\right.} \\
& \left.\quad \sqrt{\left(\sin ^{2} \phi C_{11}+\cos ^{2} \phi C_{33}\right)^{2}-4\left[\sin ^{2} \phi \cos ^{2} \phi\left(C_{11} C_{33}-C_{13}{ }^{2}\right)\right]}\right) \\
& \quad / 2 \rho]^{1 / 2}
\end{aligned}
$$

At each temperature, the three measured longitudinal sound velocities at $\varphi=0^{\circ}, 45^{\circ}$, and $90^{\circ}$ are used to calculate the three elastic constants, $C_{11}, C_{33}$, and $C_{13}$, using eq 10 . The computed $C_{11}, C_{33}$, and $C_{13}$ are shown in Figure $3 \mathrm{~b}$ as a function of temperature, covering all the $\mathrm{LC}$ states, while the results for the crystalline phase are shown in the inset. The mechanical anisotropy drops dramatically from the crystalline phase to the SmC phase. As the temperature further increases, the mechanical anisotropy decreases slightly $(0.5-2 \%)$ in the subsequent LC phases. This decreasing of the anisotropy is also found in the temperature dependency of the sound velocities at $\varphi=0^{\circ}, 45^{\circ}$, and $90^{\circ}$ in each phase (Figure 3a). In the isotropic phase, the mechanical anisotropy is negligible as expected. A compilation of the reported mechanical anisotropy data in LC phases from the literature ${ }^{5,7,8}$ is provided in Table S1.

Anisotropic Thermal Transport and Phonon MFP in the LC Phases. The anisotropic thermal transport in PYP8O8 is probed by TWA experiments. Two samples, LC-A and LC$\mathrm{B}$, are used to examine the thermal diffusivity in the $\| \mathbf{m}$ and $\perp \mathbf{m}$ directions, respectively, as shown in Figure $4 \mathrm{a}, \mathrm{b}$. The variation of $\alpha_{\|}$and $\alpha_{\perp}$ in PYP8O8 with temperature is depicted 


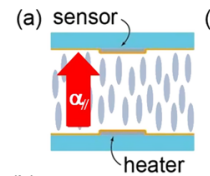

(b)

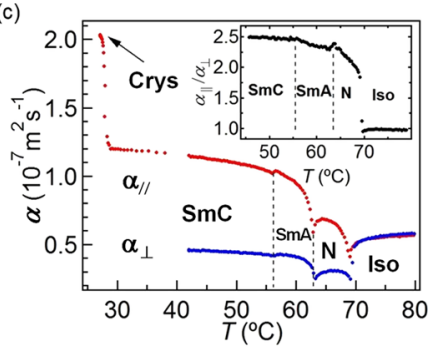

Figure 4. TWA-measured anisotropic thermal diffusivities. (a) Schematic of LC-A used to measure $\alpha_{\|}$. (b) Schematic of LC-B used to measure $\alpha_{\perp}$. (c) Temperature dependency of the two characteristic thermal diffusivities, $\alpha_{\|}$and $\alpha_{\perp}$. Inset: Temperature dependency of $\alpha_{\|} / \alpha_{\perp}$.

in Figure 4c. In all the LC phases (SmC, SmA, and N), $\alpha_{\|}$is more than twice $\alpha_{\perp}$. The temperature dependency of the thermal diffusivity ratio, $\alpha_{\|} / \alpha_{\perp}$, is shown in the inset to Figure $4 \mathrm{c}$. The ratio increases with decreasing temperature, which reflects the increases of the order parameter at low temperature. The thermal diffusivity of the polycrystalline state is also shown in Figure 4c. At the $\mathrm{SmC} \rightarrow$ Crys phase transition, the thermal diffusivity increases by about $40 \%$, which is the most significant change in all phase transitions considered in this study. The thermal diffusivity values are in the range of the non-LC polycrystalline system. ${ }^{30}$ This indicates that breaking the lattice structure is a more effective way to reduce the thermal-transport anisotropy in molecular systems than rearranging the $\mathrm{LC}$ molecules in the $\mathrm{LC}$ phases.

Based on the thermal diffusivities (Figure 4c) and sound velocities (Figure 3a), we evaluated the effective MFPs of the phonons governing the thermal transport using eq 2 . We adopted the BLS-measured sound velocities as the phonon group velocities. ${ }^{31}$ Figure 5 a depicts the temperature depend-
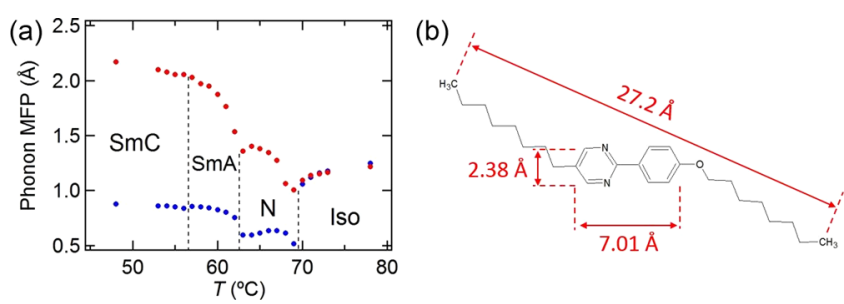

Figure 5. (a) Temperature dependence of the phonon mean free paths along (red) and perpendicular to (blue) the director of the LC molecule, estimated from the BLS-measured sound velocities and TWA-measured thermal diffusivities. (b) Molecular structure of a PYP8O8 molecule with the alkyl chains in an all-trans configuration.

encies of the phonon MFPs in the $\| \mathbf{m}$ and $\perp \mathbf{m}$ directions. In the liquid phase, the phonon MFPs are similar in both directions. Upon phase transition into a LC phase, the phonon MFP in the $\| \mathbf{m}$ direction becomes more than twice that in the $\perp \mathbf{m}$ direction (Figure 5a). The effective phonon MFPs along both directions are on the order of $1 \AA$, which is comparable to the length of a single chemical bond in the molecular structure of PYP8O8. We note that the phonon MFPs in Figure 5a underestimate the presence of long-MFP phonons, which could be better described by a thermal diffusivity accumulation function with respect to the phonon MFP..$^{23,32,33}$ The MFP of the long wavelength (low $\mathbf{q}$ ) phonons probed by BLS is about 4 orders of magnitude longer because of their long life time
(Figure S2). The results in Figure 5a provide a consistent way to qualitatively evaluate the anisotropic thermal transport in PYP8O8. To correlate the anisotropic phonon MFPs with the structural anisotropy, we show the molecular structure of PYP8O8 with the alkyl chains in an all-trans configuration (Figure $5 \mathrm{~b}$ ). The length of the chemical bond between two carbon atoms with a $\mathrm{sp}^{3}$ hybrid orbital is around $1.53 \AA$. The molecular length of PYP8O 8 in the all-trans configuration is calculated to be $27.2 \AA$, while the width is about $2.38 \AA$. The molecular length corresponds to the distance between the two carbon atoms at the free ends of the two side chains of PYP8O8, and that the width to the distance between the two carbon atoms at the 4,6 positions in the pyrimidine structure. These length and width give a structural aspect ratio of 11.4, which is much larger than the phonon MFP ratio $(\sim 2.5$, at 43 ${ }^{\circ} \mathrm{C}$ in the SmC phase).

Surprisingly, the phonon MFP ratio is close to the structural aspect ratio $(\sim 2.9)$ of the rigid core of the PYP8O8 molecule. The length of the rigid core (7.01 $\AA$, Figure $5 b)$ is defined as the distance between the two carbon atoms at both ends of the phenyl pyrimidine structure. This suggests that the phonon MFP anisotropy is dominated by the structural aspect ratio of the rigid core. In fact, it has been demonstrated that the thermal anisotropy of LCs (at least in the SmC phase) is decisively determined by the molecular anisotropy, and is independent of the long-range order. ${ }^{12}$ For thermal transport, the alkyl side chains could be treated as an intramolecular medium, where the rigid cores of the LC molecules are embedded. While the phonon MFPs could be high in the rigid core, they are typically small in the side chains because of the many scattering processes. To achieve highly thermally anisotropic LCs, it is crucial to enhance the anisotropy in the phonon MFP, which can be realized by increasing the structural anisotropy of the rigid core and reducing the length of the soft side chains.

\section{CONCLUSIONS}

The direction-dependent sound velocity and thermal diffusivity of a phenyl pyrimidine LC (PYP8O8) were examined over a wide temperature range by using BLS and TWA, respectively. As temperature increases, the LC films undergo the following phase sequence: Crys-29-SmC-56-SmA-62-N-69-Iso. Concomitantly, both the mechanical and thermal anisotropies decrease because of the reduced order parameter but the biggest changes occur at the Crys-SmC phase transition. The effective phonon MFPs in the directions parallel and perpendicular to the LC director are obtained using the experimental thermal diffusivities and sound velocities. The effective phonon MFPs in PYP8O8 are much smaller than the molecular length and width. The phonon MFP anisotropy is much smaller than the structural anisotropy of the LC molecule, but correlates with the structural anisotropy of the rigid core of the LC molecule. Increasing the structural anisotropy of the rigid core appears to be an effective way to enhance the thermoelastic anisotropy of LC samples. The determination of direction-dependent phonon MFPs in soft materials enabled by combining BLS and TWA offers new insights into thermal management via structure-based phonon engineering.

\section{ASSOCIATED CONTENT}

\section{S Supporting Information}

The Supporting Information is available free of charge on the ACS Publications website at DOI: 10.1021/acs.jpcc.9b04270. 
Elastic anisotropy of similar LCs from previous studies, BLS measurements of LC-B, and MFPs of BLS-probed phonons (PDF)

\section{AUTHOR INFORMATION}

\section{Corresponding Authors}

*E-mail: fytas@mpip-mainz.mpg.de (G.F.).

*E-mail: morikawa.j.aa@m.titech.ac.jp (J.M.).

ORCID

Junko Morikawa: 0000-0002-9530-9478

\section{Author Contributions}

The manuscript was written through contributions of all authors. All authors have given approval to the final version of the manuscript.

\section{Notes}

The authors declare no competing financial interest.

\section{ACKNOWLEDGMENTS}

Y.C., Z.W., and G.F. acknowledge the financial support by ERC AdG SmartPhon (grant no. 694977). M.R. thanks G.F. for the hospitality in MPIP and acknowledges JSPS Kakenhi (grant no. 18J14350). J.M. acknowledges the partial support by a JSPS Kakenhi (grant no. 18H04506), and JST CREST (grant no. JPMJCR18I7).

\section{REFERENCES}

(1) Andrienko, D. Introduction to liquid crystals. J. Mol. Liq. 2018, 267, 520-541.

(2) Lagerwall, J. P. F.; Scalia, G. A new era for liquid crystal research: Applications of liquid crystals in soft matter nano-, bio- and microtechnology. Curr. Appl. Phys. 2012, 12, 1387-1412.

(3) Schadt, M. Milestone in the history of field-effect liquid crystal displays and materials. Jpn. J. Appl. Phys. 2009, 48, 03B001.

(4) Liao, Y.; Clark, N. A.; Pershan, P. S. Brillouin Scattering from Smectic Liquid Crystals. Phys. Rev. Lett. 1973, 30, 639-641.

(5) Miyano, K.; Ketterson, J. B. Ultrasonic study of liquid crystals. Phys. Rev. 1975, 12, 615-635.

(6) Bradberry, G. W.; Vaughan, J. M. Brillouin scattering in the smectic A and isotropic phases of a bulk liquid crystal. J. Phys. C: Solid State Phys. 1976, 9, 3905-3910.

(7) Bradberry, G. W.; Clarke, C. F. Temperature dependence and asymmetry of the hypersound velocity in cyanobiphenyl liquid crystals. Phys. Lett. A 1983, 95, 305-308.

(8) Gleed, D. G.; Sambles, J. R.; Bradberry, G. W. Brillouin scattering in the smectic-A, nematic and isotropic phases of $4^{\prime}-\mathrm{n}$ octyloxy-4-cyanobiphenyl (8OCB). Phys. Lett. A 1989, 134, 440-444.

(9) Grammes, C.; Kriiger, J. K.; Bohn, K. P.; Bailer, J.; Fischer, C.; Schorr, C.; Rogez, D.; Alnot, P. Universal relaxation behavior of classical liquid crystals at hypersonic frequencies. Phys. Rev. E: Stat. Phys., Plasmas, Fluids, Relat. Interdiscip. Top. 1995, 51, 430-440.

(10) Krüger, J. K.; Grammes, C.; Jiménez, R.; Schreiber, J.; Bohn, K.P.; Baller, J.; Fischer, C.; Rogez, D.; Schorr, C.; Alnot, P. Secondorder elasticity of liquid crystals within their nematic state at high frequencies. Phys. Rev. E: Stat. Phys., Plasmas, Fluids, Relat. Interdiscip. Top. 1995, 51, 2115-2128.

(11) Kužel, P.; Dugautier, C.; Moch, P.; Pavel, M. Brillouin scattering in a ferroelectric liquid crystal: A study of the liquidsmectic a-smectic $C^{*}$ phase sequence. Ferroelectrics 1996, 185, 7780.

(12) Urbach, W.; Hervet, H.; Rondelez, F. Thermal diffusivity in mesophases: A systematic study in 4-4'-di-(n-alkoxy) azoxy benzenes. J. Chem. Phys. 1983, 78, 5113-5124.

(13) Rondelez, F.; Urbach, W.; Hervet, H. Origin of Thermal Conductivity Anisotropy in Liquid Crystalline Phases. Phys. Rev. Lett. 1978, 41, 1058-1062.
(14) Ahlers, G.; Cannell, D. S.; Berge, L. I.; Sakurai, S. Thermal conductivity of the nematic liquid crystal 4-n-pentyl-4'-cyanobiphenyl. Phys. Rev. E: Stat. Phys., Plasmas, Fluids, Relat. Interdiscip. Top. 1994, 49, 545-553.

(15) Marinelli, M.; Mercuri, F.; Zammit, U.; Scudieri, F. Thermal conductivity and thermal diffusivity of the cyanobiphenyl(nCB)homologous series. Phys. Rev. E: Stat. Phys., Plasmas, Fluids, Relat. Interdiscip. Top. 1998, 58, 5860-5866.

(16) Uehara, M.; Takezoe, H.; Vaupotič, N.; Pociecha, D.; Gorecka, E.; Aoki, Y.; Morikawa, J. Thermal diffusivity anisotropy measured by a temperature wave method in the homologous series of ( $\mathrm{p}$ alkoxybenzylidene)-p'-octylaniline (nO.8). J. Chem. Phys. 2015, 143, 074903.

(17) Sugimoto, A.; Yoshioka, Y.; Kang, S.; Tokita, M. Thermal diffusivity of side-chain-polymer smectic liquid crystals. Polymer 2016, $106,35-42$.

(18) Morikawa, J.; Hashimoto, T. Thermal diffusivity of aromatic polyimide thin films by temperature wave analysis. J. Appl. Phys. 2009, $105,113506$.

(19) Morikawa, J.; Hashimoto, T.; Kishi, A.; Shinoda, Y.; Ema, K.; Takezoe, H. Critical anomalies in thermal diffusivity of liquidcrystalline terephthal-bis-(4-n-butylaniline). Phys. Rev. E: Stat., Nonlinear, Soft Matter Phys. 2013, 87, 022501.

(20) Uehara, M.; Aya, S.; Araoka, F.; Ishikawa, K.; Takezoe, H.; Morikawa, J. Discontinuous Thermal Diffusivity Change due to the Anchoring Transition of a Liquid Crystal on a Perfluoropolymer Surface. ChemPhysChem 2014, 15, 1452.

(21) Ryu, M.; Takezoe, H.; Haba, O.; Yonetake, K.; Morikawa, J. Photo-controllable thermal diffusivity and thermal conductivity driven by the orientation change of nematic liquid crystal with azodendrimers. Appl. Phys. Lett. 2015, 107, 221901.

(22) Ryu, M.; Morikawa, J. Simultaneous measurements of anisotropic thermal diffusivity and thermal effusivity of liquid crystals using temperature wave analysis method. Jpn. J. Appl. Phys. 2016, 55, 111701.

(23) Yang, F.; Dames, C. Mean free path spectra as a tool to understand thermal conductivity in bulk and nanostructures. Phys. Rev. B: Condens. Matter Mater. Phys. 2013, 87, 035437.

(24) Priadilova, O.; Cheng, W.; Tommaseo, G.; Steffen, W.; Gutmann, J. S.; Fytas, G. Probing the Micromechanical Behavior of Semicrystalline Polypropylene Films by Brillouin Spectroscopy. Macromolecules 2005, 38, 2321-2326.

(25) Still, T. High Frequency Acoustics in Colloid-Based Meso- and Nanostructures by Spontaneous Brillouin Light Scattering; SpringerVerlag Berlin Heidelberg, 2010.

(26) Voudouris, P.; Gomopoulos, N.; Le Grand, A.; Hadjichristidis, N.; Floudas, G.; Ediger, M. D.; Fytas, G. Does Brillouin light scattering probe the primary glass transition process at temperatures well above glass transition? J. Chem. Phys. 2010, 132, 074906.

(27) Cusack, S.; Miller, A. Determination of the elastic constants of collagen by Brillouin light scattering. J. Mol. Biol. 1979, 135, 39-51.

(28) Kiefer, R.; Baur, G. Density studies on various smectic liquid crystals. Liq. Cryst. 1990, 7, 815-837.

(29) Ting, T. C. T.; Chen, T. Poisson's ratio for anisotropic elastic materials can have no bounds. Q. J. Mech. Appl. Math. 2005, 58, 7382.

(30) Morikawa, J.; Hashimoto, T. Simultaneous measurement of heat capacity and thermal diffusivity in solid-solid and solid-liquid phase transitions of n-alkane. Thermochim. Acta 2000, 352-353, 291-296.

(31) Schechtel, E.; Yan, Y.; Xu, X.; Cang, Y.; Tremel, W.; Wang, Z.; Li, B.; Fytas, G. Elastic Modulus and Thermal Conductivity of Thiolene/TiO2 Nanocomposites. J. Phys. Chem. C 2017, 121, 25568-25575.

(32) Henry, A. S.; Chen, G. Spectral Phonon Transport Properties of Silicon Based on Molecular Dynamics Simulations and Lattice Dynamics. J. Comput. Theor. Nanosci. 2008, 5, 141-152.

(33) Bolmatov, D.; Zhernenkov, M.; Sharpnack, L.; Agra-Kooijman, D. M.; Kumar, S.; Suvorov, A.; Pindak, R.; Cai, Y. Q.; Cunsolo, A. 
Emergent Optical Phononic Modes upon Nanoscale Mesogenic Phase

Transitions. Nano Lett. 2017, 17, 3870-3876. 